EPiC Series in Computing
Volume 58, 2019, Pages 247-256
Proceedings of 34th International Confer-
ence on Computers and Their Applications

\title{
Enhanced Prediction Models for Predicting Spatial Visualization (VZ) in Address Verification Task
}

\author{
Thitivatr PatanasakPinyo ${ }^{1}$, Georgi Batinov², Kofi Whitney ${ }^{2}$, Adel Sulaiman², \\ and Les Miller ${ }^{2}$ \\ 1 Faculty of Information and Communication Technology, Mahidol University \\ Salaya, Nakhon Pathom, 73170, Thailand \\ thitivatr.pat@mahidol.edu \\ 2 Department of Computer Science, Iowa State University \\ Ames, Iowa, 50011, USA \\ (batinov, kwhitney, aadel, lmiller)@iastate.edu
}

\begin{abstract}
In the field of Human Computer Interaction and Psychology, it is accepted that spatial visualization (VZ) is one ability that can indicate individual's performance on computer applications. Since users with different levels of VZ seem to prefer different types of user interfaces (UI), knowing a user's level of VZ provides a great opportunity for application developers to design software with higher satisfaction and usability. In this paper, we proposed three models to predict a participant's level of VZ based on the participant's actions (taps) on the tablet screen while doing an address verification task in the neighborhood using the tablet. After applying the proposed prediction models with data of thirty participants, they yielded an optimal accuracy of $93.33 \%$.
\end{abstract}

\section{Introduction}

Spatial visualization (VZ) was defined by Salthouse et al. [19] as "mental manipulation of spatial information to determine how a given spatial configuration would appear if portions of that configuration were to be rotated, folded, repositioned or otherwise transformed" [Salthouse, Timothy A., et al. "Sources of individual differences in spatial visualization ability." Intelligence 14.2 (1990): 187-230]. It has been known to be an ability that determines usage performance of a user [19]. For example (but not limited to), the time a user uses to understand a UI of a software application. Another fact about VZ is that people with different levels of VZ prefer different types of UI to work with $[1,14,15,17]$. Therefore, aiming at optimal usage performance requires an application to provide different UIs that fit each different VZ level, which is basically the idea of adaptive UI application [3]. Since asking users to complete the VZ test, e.g., Paper Folding Test (VZ-2) [8], every time they use the application (to assess their VZ level) is not practical, predicting the VZ level from a user's interaction with a UI in real-time is one feasible answer. We proposed these new prediction models for predicting a user's level of VZ while he/she is doing an address verification task in the actual neighborhood,

G. Lee and Y. Jin (eds.), CATA 2019 (EPiC Series in Computing, vol. 58), pp. 247-256 
using a location-based software application on a tablet. The main contribution of this paper is the development of a new feature set for VZ prediction in address verification software. The new feature set allowed us to predict the VZ category of participants at a higher rate than was shown in Batinov [2]. To test the power of the new feature set, we compared it to two other feature sets derived from Batinov [2].

We organized this paper as follows: Section 2 examines related literature. Section 3 describes the methodology that we used to design and implement the models. Section 4 shows the improvement of accuracy when we executed the new models with some discussion. Finally, Section 5 summarizes the contribution described in this paper.

\section{Related Work}

VZ is one ability that belongs to the set of human spatial ability. Several scientists discovered that VZ provides an indicator of the performance of a user using computer software $[4,7,12]$. Other studies also show that VZ might be used to predict the level of success a user has while using software [10,11]. Furthermore, it was also known that users with a high VZ level tend to perform better than users with low VZ level when it comes to software usage performance [6]. Batinov et al. showed that the effect of VZ also holds in the task of address verification [1].

Batinov [2] proposed a prediction model that can predict an individual's level of VZ (either high or low). We built on his work in our proposed work, which we describe in Section 3.

\section{Methodology}

Batinov [2] created a prediction model that predicts an individual's level of VZ using the actions that the user performed while using the application. We denote Batinov's model by $\mathbb{I}_{0}$. In his study, he asked participants to verify addressing units whether or not they were located in the correct location. A participant was equipped with the location-based software application that contained a map of focused neighborhood, a list of addresses to be verified, and necessary map operations, e.g., pan \& zoom. Every time a participant performed an action on application UI, the action was logged. He then extracted data from the log file to create instances for $\mathbb{M}_{0}$.

In $\mathbb{M}_{0}$, a set of features of each participant, denoted by $\mathbb{F}\left(\mathbb{M}_{0}\right)$, consists of twenty-two features and one class attribute. Particularly, for each participant, $\mathbb{F}\left(\mathbb{M}_{0}\right)$ contains six numbers he/she specifically changed a focus to addresses 1, 2, 3, 4, 5, or 6, respectively (Note that Batinov had six addresses in his study), a number he/she changed a focus to any address, a number he/she clicked zoom-in icon, a number he/she reset the map, a number he/she panned up, a number he/she clicked zoom-out icon, a number he/she attempted to pan beyond the map, a number he/she did 1-level zoom out by slider, a number he/she clicked center zoom, a number he/she panned right, a number he/she panned left, a number he/she panned down, a number he/she did 3-level zoom in by slider, a number he/she did 1-level zoom in by slider, a number he/she did 2-level zoom out by slider, a number he/she did 2-level zoom in by slider, a number he/she did 3-level zoom out by slider, and a class attribute. The class attribute specifies the actual level of VZ (high/low). The classifier that was implemented is Bagging [5]. The base classifier of Bagging is REPTree, which is a default base classifier for Bagging in Weka.

We applied $\mathbb{M}_{0}$ to our data we gathered during the field study to observe the accuracy of prediction. Particularly, our study was also an address verification. We asked thirty participants to verify ten addresses in the neighborhood near the central campus of Iowa State University using Android tablet [14]. The study was divided into two phases. A difference between 
both phases was a type of UI of the software application. Every participant was randomly assigned either traditional UI or adaptive UI to be used for verification in the first phase. If the traditional UI was selected for the first phase, the adaptive UI then was assigned for the second phase and vice versa. The traditional UI was a UI that contained only necessary tools such as zoom buttons, pan buttons, real-time location (GPS), Object-Indexing [16], and etc. The adaptive UI was a UI that contained both necessary tools and additional UI features that were evidently useful for participants with different levels of VZ. An example of those UI features was a landmark on the map, which increased usage performance of participants whose VZ level was low $[13,18,21]$. Figure 1 shows the application UI in our study (traditional UI).

After applying $\mathbb{M}_{0}$ with data set gathered from our study, we retrieved the prediction accuracy of $83 \%$. Although $83 \%$ is highly acceptable, we decided to improve the model further with the goal to maximize prediction accuracy as much as possible. We came up with three new prediction models: Prediction Models I, II, and III, which are denoted by $\mathbb{I M}_{1}, \mathbb{M}_{2}$, and $\mathrm{M}_{3}$, respectively.

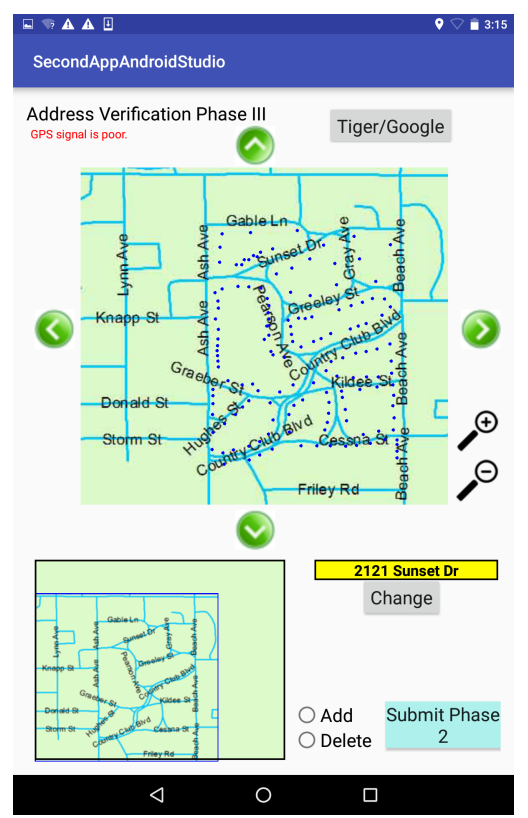

Figure 1: Application UI (Traditional).

\subsection{Prediction Model I $\left(\mathrm{M}_{1}\right)$}

For the first model, $\mathbb{M}_{1}$, we replaced the base classifier of Bagging with J48 rather than REPTree. We defined a new set of features, denoted by $\mathbb{F}\left(\mathbb{M}_{1}\right)$, as $\mathbb{F}\left(\mathbb{M}_{1}\right)=$ $\{T T, E T, P T, Z T, I O, O I, L R, R L, U D, D U, T R, V Z\}$. Table 1 describes $\mathbb{F}\left(\mathbb{M}_{1}\right)$.

$\mathbb{F}\left(\mathbb{M}_{1}\right)$ consists of eleven features and one class attribute. TT stores a number of total taps that a participant made on the application UI. ET stores a number of error taps that a participant made, particularly, an error tap is a tap on any non-sensitive region [20] of UI. PT and $Z T$ store numbers of pans (left, right, up, or down) and zooms (in or out), respectively. Next six features $(I O, O I, L R, R L, U D$, and $D U)$ represents numbers of reversals that a 


\begin{tabular}{||cc||}
\hline Feature & Description \\
\hline \hline$T T$ & Total taps a participant made on UI \\
\hline$E T$ & Total error taps a participant made on UI \\
\hline$P T$ & Total pans a participant panned the map \\
\hline$Z T$ & Total zooms a participant zoomed the map \\
\hline$I O$ & Total in-out reversals a participant made \\
\hline$O I$ & Total out-in reversals a participant made \\
\hline$L R$ & Total left-right reversals a participant made \\
\hline$R L$ & Total right-left reversals a participant made \\
\hline$U D$ & Total up-down reversals a participant made \\
\hline$D U$ & Total down-up reversals a participant made \\
\hline$T R$ & Total reversals a participant made on UI \\
\hline$V Z$ & Level of VZ \\
\hline
\end{tabular}

Table 1: A Set of Features of $\mathbb{M}_{1}\left(\mathbb{F}\left(\mathbb{M}_{1}\right)\right)$.

participant made. A reversal is a series of map operations that, finally, yields the same result as the original map before performing those operations [1]. For instance, a participant pans right and pans left the map, which results the same map area before panning. $I O$ stores a number of in-out reversals, which is a reversal caused by zooming in the map followed by zooming out the map. Similarly for $O I$, it stores a number of out-in reversals. $L R, R L, U D$, and $D U$ focus on reversals by panning. $L R$ stores a number of left-right reversals, which caused by panning left followed by panning right. Similarly, $R L, U D$, and $D U$ store numbers of right-left reversals, up-down reversals, and down-up reversals, respectively. The last one, $V Z$, is the class attribute specifying level of VZ (low/high).

\subsection{Prediction Model II $\left(\mathbb{M}_{2}\right)$}

The second model, $\mathbb{M}_{2}$, inherited classifier and base classifier from $\mathbb{M}_{1}$, which are Bagging with $\mathrm{J} 48$ as base classifier, respectively. We defined a set of features for $\mathrm{M}_{2}\left(\mathbb{F}\left(\mathbb{M}_{2}\right)\right)$ as $\mathbb{F}\left(\mathbb{M}_{2}\right)=$ $\left\{T T, E T, P T, Z T, I O, O I, L R, R L, U D, D U, T R,+,-,<,>, A, V, Z, H O R_{1}, H O R_{2}, V Z\right\}$. Table 2 describes $\mathbb{F}\left(\mathbb{M}_{2}\right)$.

For $\mathbb{F}\left(\mathrm{M}_{2}\right)$, it contains twenty features and one class attribute. First eleven attributes $(T T, E T, P T, Z T, I O, O I, L R, R L, U D, D U, T R)$ are borrowed from $\mathbb{F}\left(\mathbb{M}_{1}\right)$. Furthermore, we added seven more features that we extracted from $\mathbb{M}_{0}$ into $\mathbb{F}\left(\mathbb{M}_{2}\right)$. Those seven features are $+,-,<,>, A, V$, and $Z$. + represents how many times a participant tapped zoom-in button to zoom in the map. - represents how many times a participant tapped zoom-out button to zoom out the map. < represents how many times a participant tapped pan-left button to pan left the map. $>$ represents how many times a participant tapped pan-right button to pan right the map. A represents how many times a participant tapped pan-up button to pan up the map. $V$ represents how many times a participant tapped pan-up button to pan up the map. $Z$ represents how many times a participant changed a working address. Since there are ten addresses that a participant needed to verify, he/she could arbitrarily switch to any address on the fly. However, there are two features that we added to $\mathbb{F}\left(\mathrm{M}_{2}\right)$, which are $H O R_{1}$ and $H_{O} R_{2}$. $H O R_{1}$ and $H O R_{2}$ store numbers of high-order reversal - degree 2 in Phases 1 and 2 of the study, respectively. A high-order reversal is a special case of reversal where a series of map operations contains more than one pair of reversal without any other tap is done in between. 


\begin{tabular}{||cc||}
\hline Feature & Description \\
\hline \hline$T T$ & Total taps a participant made on UI \\
\hline$E T$ & Total error taps a participant made on UI \\
\hline$P T$ & Total pans a participant panned the map \\
\hline$Z T$ & Total zooms a participant zoomed the map \\
\hline$I O$ & Total in-out reversals a participant made \\
\hline$O I$ & Total out-in reversals a participant made \\
\hline$L R$ & Total left-right reversals a participant made \\
\hline$R L$ & Total right-left reversals a participant made \\
\hline$U D$ & Total up-down reversals a participant made \\
\hline$D U$ & Total down-up reversals a participant made \\
\hline$T R$ & Total reversals a participant made on UI \\
\hline+ & Total times a participant zoomed in \\
\hline- & Total times a participant zoomed out \\
\hline$<$ & Total times a participant panned left \\
\hline$>$ & Total times a participant panned right \\
\hline$A$ & Total times a participant panned up \\
\hline$V$ & Total times a participant panned down \\
\hline$Z$ & Total times a participant changed address \\
\hline$H O R_{1}$ & Total high-order reversals in Phase I \\
\hline$H O R_{2}$ & Total high-order reversals in Phase II \\
\hline$V Z$ & Level of VZ \\
\hline
\end{tabular}

Table 2: A Set of Features of $\mathbb{M}_{2}\left(\mathbb{F}\left(\mathbb{M}_{2}\right)\right)$.

Previously in $\mathbb{M}_{1}$, we focused on a reversal that consisted of exactly two map operations, for example, pan up \& pan down or zoom in \& zoom out. However, after taking more closer look at $\log$ file of every participant, we found an interesting pattern. This pattern was a series of two pairs (four map operations) of reversal such that any single map operations in the series can be arranged in any permutation as long as the series preserved reversal property, i.e., a result after performing all those map operations in the series is the same as the original map before performing. We named this pattern as high-order reversal - degree $\mathbf{2}$ since it contained two pairs of reversal. Examples of high-order reversal - degree 2 are \{pan-up, pan-down, zoom-in, zoom-out $\}$ or (pan-left, pan-left, pan-right, pan-right). For the latter, two map operations from the same pair of reversal do not need to be contiguous to each other, e.g., pan-right does not need to follow pan-left. The last attribute, $V Z$, is the class attribute specifying level of VZ.

\subsection{Prediction Model III $\left(\mathbb{M}_{3}\right)$}

After we have got two prediction models, $\mathrm{M}_{1}$ and $\mathbb{M}_{2}$, we would like to see how the additional features that we added to $\mathbb{F}\left(\mathbb{M}_{2}\right)$ affected the prediction accuracy. Therefore, we created the last prediction model, denoted as $\mathbb{M}_{3}$, such that $\mathbb{F}\left(\mathbb{M}_{3}\right)=\mathbb{F}\left(\mathbb{M}_{2}\right) \backslash \mathbb{F}\left(\mathbb{M}_{1}\right)=\{+,-,<,>$ , $\left.A, V, Z, H O R_{1}, H O R_{2}, V Z\right\}$ Hence, $\mathbb{F}\left(\mathbb{M}_{3}\right) \subset \mathbb{F}\left(\mathbb{M}_{2}\right)$. Table 3 describes $\mathbb{F}\left(\mathbb{M}_{3}\right)$.

After we designed those three models, $\mathbb{M}_{1}, \mathbb{M}_{2}$, and $\mathbb{M}_{3}$, we implemented them using Weka [9]. Results of implementation are discussed in Section 4. 


\begin{tabular}{||cc||}
\hline Feature & Description \\
\hline \hline+ & Total times a participant zoomed in \\
\hline- & Total times a participant zoomed out \\
\hline$<$ & Total times a participant panned left \\
\hline$A$ & Total times a participant panned right \\
\hline$V$ & Total times a participant panned up \\
\hline$Z$ & Total times a participant panned down \\
\hline$H O R_{1}$ & Total times a participant changed address \\
\hline$H O R_{2}$ & Total high-order reversals in Phase I \\
\hline$V Z$ & Total high-order reversals in Phase II \\
\hline
\end{tabular}

Table 3: A Set of Features of $\mathrm{M}_{3}\left(\mathbb{F}\left(\mathbb{M}_{3}\right)\right)$.

\section{Results and Discussion}

$\mathrm{M}_{0}, \mathrm{M}_{1}, \mathrm{M}_{2}$, and $\mathrm{M}_{3}$ were implemented and tested using data set from our study, which consisted of thirty instances. Each instance represents features of each participant. Every feature and class attribute are non-identifiable data with respect to the protocol of IRB [14]. For each model, the classifier was Bagging with 10 iterations and J48 as base classifier.

\subsection{Classification Results of $\mathrm{M}_{0}$}

Batinov [2] ran multiple tests to test his $\mathbb{M}_{0}$. There were twenty-two tests. For each test, it had thirty-one instances representing thirty-one participants. The difference between each test was a recessed radius when he logged (but we will not go into detail about it in this paper). The average accuracy were around $82 \%$.

Although $\mathbb{M}_{0}$ did not perfectly fit with our data due to the difference between UIs of Batinov's software [2] and ours, e.g., some features such as a number of zoom-in by slider would be 0 because our UI has no slider, we still applied $\mathbb{M}_{0}$ with our data for observation. $\mathbb{M}_{0}$ yielded an accuracy of $90 \%$ (27 correctly predicted participants). One participant who tested low VZ was classified as high VZ and two participants who tested high VZ were classified as low VZ.

\subsection{Classification Results of $\mathrm{M}_{1}$}

There were twenty-eight correctly classified instances, which was around 93.33\%. Two participants who tested low VZ were classified as high VZ. Tables 4 and 5 show the summary and confusion matrix, respectively.

\begin{tabular}{||llc||}
\hline \hline Correctly Classified Instances & 28 & $93.3333 \%$ \\
Incorrectly Classified Instances & 2 & $6.6667 \%$ \\
Kappa statistic & 0.8421 & \\
Mean absolute error & 0.1373 & \\
Root mean squared error & 0.2279 & \\
Relative absolute error & $44.337 \%$ & \\
Root relative squared error & $59.0762 \frac{8}{8}$ & \\
Total Number of Instances & 30 & \\
\hline
\end{tabular}

Table 4: Summary of $\mathbb{M}_{1}$. 


\begin{tabular}{|rllll||}
\hline \hline $\mathrm{a}$ & $\mathrm{b}$ & $\mathrm{c}$ & $<--$ classified as \\
20 & 0 & 0 & $\mathrm{|}$ & $\mathrm{a}=\mathrm{H}$ \\
2 & 8 & 0 & $\mathrm{l}$ & $\mathrm{b}=\mathrm{L}$ \\
\hline
\end{tabular}

Table 5: Confusion Matrix of $\mathbb{M}_{1}$.

\subsection{Classification Results of $\mathrm{M}_{2}$}

For evaluation on training data, there were twenty-eight correctly classified instances, which was 93.33\%. Two participants who tested low VZ were classified as high VZ. Furthermore, those misclassified two were the same participants who were incorrectly classified in $\mathbb{M}_{1}$. Tables 6 and 7 show the summary and confusion matrix, respectively, for the 30 participants.

\begin{tabular}{||llc||}
\hline \hline Correctly Classified Instances & 28 & $93.3333 \%$ \\
Incorrectly Classified Instances & 2 & $6.6667 \%$ \\
Kappa statistic & 0.8421 & \\
Mean absolute error & 0.1432 & \\
Root mean squared error & 0.2317 & \\
Relative absolute error & $46.2283 \%$ \\
Root relative squared error & $60.069 \%$ \\
Total Number of Instances & 30 \\
\hline \hline
\end{tabular}

Table 6: Summary of $\mathbb{M}_{2}$.

\begin{tabular}{|c|c|c|c|}
\hline a & $\mathrm{b}$ & $\mathrm{c}$ & <-- classified as \\
\hline 20 & 0 & 01 & $a=H$ \\
\hline 2 & 8 & $0 \quad 1$ & $\mathrm{~b}=\mathrm{L}$ \\
\hline
\end{tabular}

Table 7: Confusion Matrix of $\mathrm{IM}_{2}$.

\subsection{Classification Results of $\mathrm{M}_{3}$}

There were twenty-eight correctly classified instances, which, again, was $93.33 \%$. Tables 8 and 9 show the summary and confusion matrix, respectively. According to Table 9, there was one participant who tested low VZ but classified as high VZ. This participant was the same one who was misclassified in both $\mathrm{M}_{1}$ and $\mathbb{M}_{2}$. There was another participant who tested high $\mathrm{VZ}$ but classified as low VZ.

\begin{tabular}{||lll||}
\hline \hline Correctly Classified Instances & 28 & $93.3333 \%$ \\
Incorrectly Classified Instances & 2 & $6.6667 \%$ \\
Kappa statistic & 0.85 & \\
Mean absolute error & 0.1583 & \\
Root mean squared error & 0.2396 & \\
Relative absolute error & $51.094 \%$ \\
Root relative squared error & $62.1317 \%$ \\
Total Number of Instances & 30 \\
\hline
\end{tabular}

Table 8: Summary of $\mathbb{M}_{3}$. 


\begin{tabular}{|c|c|c|c|}
\hline a & $\mathrm{b}$ & c & <-- classified as \\
\hline 19 & 1 & 0 & $a=H$ \\
\hline 1 & 9 & 0 & $\mathrm{~b}=\mathrm{L}$ \\
\hline 0 & 0 & 0 & $c=M$ \\
\hline
\end{tabular}

Table 9: Confusion Matrix of $\mathbb{M}_{3}$.

At first, we expected to see better prediction result from $\mathbb{M}_{2}$ because $\mathbb{M}_{2}$ was the extended version of $\mathrm{IM}_{1}$. However, the actual outcome was opposite. There are potentially two causations that we were curious. One causation is that the number of our instances was not big enough. From thirty participants, there were participants that tested high VZ but his/her actual behavior was more like low VZ (and another way around). For example, one participant might test high VZ but he/she created a reversal, which is a behavior of low VZ participant when getting lost [1], multiple times in the field. Perhaps his/her intention was just to explore the UI. This incident plausibly reduces the performance of $\mathbb{M}_{2}$.

Another causation that is worth taking a look is the difference between $\mathbb{M}_{1}$ and $\mathbb{M}_{2}$. As mentioning earlier, we created a new model, $\mathbb{M}_{3}$, where $\mathbb{F}\left(\mathbb{M}_{3}\right)=\mathbb{F}\left(\mathbb{M}_{2}\right) \backslash \mathbb{F}\left(\mathbb{M}_{1}\right)$. An interesting result of $\mathbb{M}_{3}$ is that it incorrectly classified two instances. One misclassified instance was a participant who tested high VZ. Another one was a participant who tested low VZ. The result of classification of $\mathbb{M}_{3}$ was different compared to $\mathbb{M}_{1}$ and $\mathbb{M}_{2}$ in term of there existing a participant who tested high VZ but classified as low VZ, which never existed in previous two models. This misprediction was potentially caused by the same incident we have already mentioned: a participant that tested high VZ but his/her actual behavior was more like low VZ.

With this observation, a potential improvement that we would like to mark as a guideline for future work is to increase a number of instances and allow high-order reversal with degree higher than 2 to be included. Although it can affect a process of UI design such as total levels of zoom must be deeper to make a series of zoom tap relevant and significant, this guideline would help creating a new model of VZ prediction on location-based application with higher accuracy.

\section{Conclusion and Future Work}

We proposed three prediction models, $\mathrm{M}_{1}, \mathrm{M}_{2}$, and $\mathrm{IM}_{3}$, that predicted level of $\mathrm{VZ}$ of participants when they used the location-based software application to verify addresses in the neighborhood. A difference between those three is a set of features. Features of $\mathbb{M}_{1}$ consists of metrics of map operations such as total taps or total zooms. It also contains numbers of reversal pair such as left-right reversal. $\mathbb{M}_{2}$ is the extended model of $\mathbb{M}_{1}$. We added some features from Batinov's model [2] into $\mathbb{F}\left(\mathbb{M}_{2}\right)$ such as number of zoom-in. We also added numbers of high-order reversal into $\mathbb{F}\left(\mathrm{M}_{2}\right)$.

After we applied both $\mathbb{M}_{1}$ and $\mathbb{M}_{2}$ on data that we gathered from thirty participants in the address verification task using location-based software application, they returned same accuracy rate, which is $93.33 \%$. 93.33\% was the first contribution of our proposed work since we are not aware of a prediction model that could predict VZ level of user from user's set of map operations and yielded more than $90 \%$ accuracy rate.

Another contribution takes place when we created $\mathbb{M}_{3}, \mathbb{F}\left(\mathbb{M}_{3}\right)=\mathbb{F}\left(\mathbb{M}_{2}\right) \backslash \mathbb{F}\left(\mathbb{M}_{1}\right)$, to justify whether or not extra features that were added to $\mathbb{M}_{2}$ matter. The outcome showed that $\mathbb{M}_{3}$ 
also yielded the accuracy of $93.33 \%$ with dissimilar classification result.

The work described here will be incorporated into the address verification software used in [14]. The software will be used to test the impact on the workflow of the higher VZ prediction rates.

\section{References}

[1] Georgi Batinov, Michelle Rusch, Tianyu Meng, Kofi Whitney, Thitivatr Patanasakpinyo, Les Miller, and Sarah Nusser. Understanding map operations in location-based surveys. In Eighth International Conference on Advances in Computer-Human Interactions (ACHI 2015), pages 144149, Lisbon, Portugal, 2015.

[2] Georgi Iliev Batinov. Computer detection of spatial visualization in a location-based task. Graduate Theses and Dissertations: http://lib.dr.iastate.edu/etd/15103, 2017.

[3] D Benyon, PR Innocent, and D Murray. System adaptivity and the modeling of stereotypes, interact'87. In Second IFIP Conference on Human-Computer Interaction, Stuttgart, Elsevier Science Publishers, 1987.

[4] DR Benyon and DM Murray. Special issue on intelligent interface technology: editor's introduction. Interacting with Computers, 12(4):315-322, 2000.

[5] Leo Breiman. Bagging predictors. Mach. Learn., 24(2):123-140, August 1996.

[6] Thorsten Büring, Jens Gerken, and Harald Reiterer. Usability of overview-supported zooming on small screens with regard to individual differences in spatial ability. In Proceedings of the working conference on Advanced visual interfaces, pages 233-240. ACM, 2006.

[7] Dennis E Egan. Individual differences in human-computer interaction. Handbook of humancomputer interaction, pages 543-568, 1988.

[8] Ruth B Ekstrom, John W French, Harry H Harman, and Diran Dermen. Manual for kit of factor-referenced cognitive tests. Princeton, NJ: Educational testing service, 1976.

[9] Mark Hall, Eibe Frank, Geoffrey Holmes, Bernhard Pfahringer, Peter Reutemann, and Ian H Witten. The weka data mining software: an update. ACM SIGKDD explorations newsletter, 11(1):10-18, 2009.

[10] David F Lohman, James W Pellegrino, David L Alderton, and JW Regian. Dimensions and components of individual differences in spatial abilities. Intelligence and cognition: Contemporary frames of reference, pages 253-312, 1987.

[11] Mark G McGee. Human spatial abilities: Psychometric studies and environmental, genetic, hormonal, and neurological influences. Psychological bulletin, 86(5):889, 1979.

[12] Kent L Norman. Spatial visualization - a gateway to computer-based technology. Journal of Special Education Technology, 12(3):195-206, 1994.

[13] Elizabeth M O'Laughlin and Bradley S Brubaker. Use of landmarks in cognitive mapping: Gender differences in self report versus performance. Personality and Individual Differences, 24(5):595601, 1998.

[14] Thitivatr PatanasakPinyo. Flattening methods for adaptive location-based software to user abilities. Graduate Theses and Dissertations, Iowa State University, 2017.

[15] Thitivatr PatanasakPinyo, Georgi Batinov, Kofi Whitney, and Les Miller. Methods that flatten the user space for individual differences in location-based surveys on portable devices. In 31 st International Conference on Computers and Their Applications (CATA 2016), pages 65-70, Las Vegas, Nevada, 2016. International Society for Computers and their Applications (ISCA).

[16] Thitivatr PatanasakPinyo, Georgi Batinov, Kofi Whitney, Adel Sulaiman, and Les Miller. Objectindexing: A solution to grant accessibility to a traditional raster map in location-based application to accomplish a location-based task. In 8th International Conference on Computer, Electrical, and 
Electronics Engineering and Technology (ICEEET 2018), pages 126-131, Kyoto, Japan, 2018. International Centre of Excellence in Engineering and Technology (ICEEAT).

[17] Thitivatr PatanasakPinyo, Georgi Batinov, Kofi Whitney, Adel Sulaiman, Les Miller, and Stephen Gilbert. Extracting useful features for users with different levels of spatial visualization. In 33rd International Conference on Computers and Their Applications (CATA 2018), pages 86-91, Las Vegas, Nevada, 2018. International Society for Computers and their Applications (ISCA).

[18] Martin Raubal and Stephan Winter. Enriching wayfinding instructions with local landmarks. In International Conference on Geographic Information Science, pages 243-259. Springer, 2002.

[19] Timothy A Salthouse, Renee L Babcock, Debora RD Mitchell, Roni Palmon, and Eric Skovronek. Sources of individual differences in spatial visualization ability. Intelligence, 14(2):187-230, 1990.

[20] Alfred Taylor Sr, Les Miller, Sree Nilakanta, Jeffry Sander, Saayan Mitra, Anurag Sharda, and Bachar Chama. Using an error detection strategy for improving web accessibility for older adults. In Advances in Computer-Human Interactions, 2009. ACHI'09. Second International Conferences on, pages 375-380. IEEE, 2009.

[21] Michael Tlauka and Paul N Wilson. The effect of landmarks on route-learning in a computersimulated environment. Journal of Environmental Psychology, 14(4):305-313, 1994. 